\title{
An Improved Iterative Method for Solving a Class of Coupled Conductive-Radiative Heat-Transfer Problems
}

\author{
C. E. Siewert \\ Center for Research in Scientific Computation \\ Mathematics Department \\ North Carolina State University \\ Raleigh, NC 27695-8205
}

USA

\begin{abstract}
Newton's method of iteration is used along with the the $P_{N}$ method, also called the spherical-harmonics method, and Hermite cubic splines to develop an iterative solution to a class of nonlinear problems in radiative transfer. Anisotropic scattering and specularly and diffusely reflecting boundaries are allowed for the steady-state, combined-mode, conductive-radiative, heat-transfer problem considered.
\end{abstract}




\section{Introduction}

In a recent paper, Siewert and Thomas ${ }^{1}$ used a computationally stable version of the $P_{N}$ method ${ }^{2}$ and Hermite cubic splines to solve a steady-state problem in combined mode, conduction and radiation, heat transfer that was formulated by Özışık. ${ }^{3}$ Although in Ref. 1, some good numerical results were obtained from a method based on a simple iterative procedure, the authors mentioned that there were cases where that iteration scheme failed (in a very spectacular manner). Here we follow some recent work of Kelley ${ }^{4}$ and use Newton's method to define our method of iteration. Since this work is, except for the method of iteration, very similar to Ref. 1, we assume that Ref. 1 is available, and so our presentation here is brief.

We consider the equation of transfer written ${ }^{3}$ as

$$
\mu \frac{\partial}{\partial \tau} I(\tau, \mu)+I(\tau, \mu)=\frac{\varpi}{2} \sum_{l=0}^{L} \beta_{l} P_{l}(\mu) \int_{-1}^{1} P_{l}\left(\mu^{\prime}\right) I\left(\tau, \mu^{\prime}\right) d \mu^{\prime}+(1-\varpi) \frac{\sigma n^{2}}{\pi} T^{4}(\tau),
$$

for $\tau \in\left(0, \tau_{0}\right)$ and $\mu \in[-1,1]$, and the boundary conditions

$$
I(0, \mu)=\epsilon_{1} \frac{\sigma n^{2}}{\pi} T_{1}^{4}+\rho_{1}^{s} I(0,-\mu)+2 \rho_{1}^{d} \int_{0}^{1} I\left(0,-\mu^{\prime}\right) \mu^{\prime} d \mu^{\prime}
$$

and

$$
I\left(\tau_{0},-\mu\right)=\epsilon_{2} \frac{\sigma n^{2}}{\pi} T_{2}^{4}+\rho_{2}^{s} I\left(\tau_{0}, \mu\right)+2 \rho_{2}^{d} \int_{0}^{1} I\left(\tau_{0}, \mu^{\prime}\right) \mu^{\prime} d \mu^{\prime}
$$

for $\mu \in[0,1]$. Here $\tau \in\left[0, \tau_{0}\right]$ is the optical variable, $\mu$ is the direction cosine measured from the positive $\tau$ axis and $\varpi$ is the albedo for single scattering. In addition, we have assumed that the scattering law $p(\Theta)$ can be represented by a finite Legendre expansion in terms of the cosine of the scattering angle $\Theta$, i.e.

$$
p(\Theta)=\sum_{l=0}^{L} \beta_{l} P_{l}(\cos \Theta)
$$

where $\beta_{0}=1$ and $\left|\beta_{l}\right|<2 l+1$ for $l \geq 1$. In regard to the boundary conditions, we note that $\rho_{\alpha}^{s}$ and $\rho_{\alpha}^{d}$, for $\alpha=1$ and 2, are coefficients for specular and diffuse reflection and that $\epsilon_{\alpha}=1-\rho_{\alpha}^{s}-\rho_{\alpha}^{d}, \alpha=1$ and 2 , are the emissivities for the two surfaces. In addition $n$ is the index of refraction and $\sigma$ is the Stefan-Boltzmann constant.

The nonlinear aspect of this problem comes from the fact that the temperature distribution $T(\tau)$ appearing in Eq. (1) must satisfy the heat conduction equation ${ }^{3}$

$$
k \beta \frac{d^{2}}{d \tau^{2}} T(\tau)=\frac{d}{d \tau} q_{r}(\tau)
$$

subject to the boundary conditions $T(0)=T_{1}$ and $T\left(\tau_{0}\right)=T_{2}$, where $T_{1}$ and $T_{2}$ are the temperatures that also appear in Eqs. (2). In addition, $\mathrm{k}$ is the thermal conductivity of the medium, $\beta$ is the extinction 
coefficient and $q_{r}(\tau)$ is the radiative heat flux, i.e.

$$
q_{r}(\tau)=2 \pi \int_{-1}^{1} I\left(\tau, \mu^{\prime}\right) \mu^{\prime} d \mu^{\prime}
$$

\section{Basic Development}

To follow a tradition in the heat transfer literature, ${ }^{3}$ we normalize the considered problem by introducing a convenient reference temperature $T_{r}$ and by using

$$
\begin{aligned}
I(\tau, \mu) & =\left(\frac{\sigma n^{2}}{\pi} T_{r}^{4}\right) I^{*}(\tau, \mu), \\
q_{r}(\tau) & =\left(\frac{\sigma n^{2}}{\pi} T_{r}^{4}\right) q_{r}^{*}(\tau)
\end{aligned}
$$

and

$$
T(\tau)=T_{r} \Theta(\tau)
$$

to rewrite our problem as

$$
\mu \frac{\partial}{\partial \tau} I^{*}(\tau, \mu)+I^{*}(\tau, \mu)=\frac{\varpi}{2} \sum_{l=0}^{L} \beta_{l} P_{l}(\mu) \int_{-1}^{1} P_{l}\left(\mu^{\prime}\right) I^{*}\left(\tau, \mu^{\prime}\right) d \mu^{\prime}+(1-\varpi) \Theta^{4}(\tau),
$$

for $\tau \in\left(0, \tau_{0}\right)$ and $\mu \in[-1,1]$,

$$
I^{*}(0, \mu)=\epsilon_{1} \Theta_{1}^{4}+\rho_{1}^{s} I^{*}(0,-\mu)+2 \rho_{1}^{d} \int_{0}^{1} I^{*}\left(0,-\mu^{\prime}\right) \mu^{\prime} d \mu^{\prime}
$$

and

$$
I^{*}\left(\tau_{0},-\mu\right)=\epsilon_{2} \Theta_{2}^{4}+\rho_{2}^{s} I^{*}\left(\tau_{0}, \mu\right)+2 \rho_{2}^{d} \int_{0}^{1} I^{*}\left(\tau_{0}, \mu^{\prime}\right) \mu^{\prime} d \mu^{\prime}
$$

for $\mu \in[0,1]$. In addition

$$
\frac{d^{2}}{d \tau^{2}} \Theta(\tau)=\frac{1}{4 \pi N_{c}} \frac{d}{d \tau} q_{r}^{*}(\tau)
$$

with

$$
\begin{aligned}
& \Theta(0)=\Theta_{1}=\frac{T_{1}}{T_{r}}, \\
& \Theta\left(\tau_{0}\right)=\Theta_{2}=\frac{T_{2}}{T_{r}}
\end{aligned}
$$

and

$$
q_{r}^{*}(\tau)=2 \pi \int_{-1}^{1} I^{*}\left(\tau, \mu^{\prime}\right) \mu^{\prime} d \mu^{\prime}
$$

Here

$$
N_{c}=\frac{k \beta}{4 \sigma n^{2} T_{r}^{3}}
$$


is called the conduction-to-radiation parameter. ${ }^{3}$

At this point, we choose to express the inhomogeneous source term

$$
S(\tau)=(1-\varpi) \Theta^{4}(\tau)
$$

as

$$
S(\tau)=\sum_{k=0}^{K} a_{k} \phi_{k}\left(\tau / \tau_{0}\right)
$$

where we consider $K$ to be odd and that the spline functions $\left\{\phi_{k}(x)\right\}$ are as used in Ref. 1. Here, the coefficients $\left\{a_{k}\right\}$ are to be determined. It follows that we can write

$$
I^{*}(\tau, \mu)=I_{h}^{*}(\tau, \mu)+\sum_{k=0}^{K} a_{k} I_{k}^{*}(\tau, \mu)
$$

where the basis problems, for $k=0,1,2, \ldots, K$, are defined by

$$
\mu \frac{\partial}{\partial \tau} I_{k}^{*}(\tau, \mu)+I_{k}^{*}(\tau, \mu)=\frac{\varpi}{2} \sum_{l=0}^{L} \beta_{l} P_{l}(\mu) \int_{-1}^{1} P_{l}\left(\mu^{\prime}\right) I_{k}^{*}\left(\tau, \mu^{\prime}\right) d \mu^{\prime}+\phi_{k}\left(\tau / \tau_{0}\right),
$$

for $\tau \in\left(0, \tau_{0}\right)$ and $\mu \in[-1,1]$,

$$
I_{k}^{*}(0, \mu)=\rho_{1}^{s} I_{k}^{*}(0,-\mu)+2 \rho_{1}^{d} \int_{0}^{1} I_{k}^{*}\left(0,-\mu^{\prime}\right) \mu^{\prime} d \mu^{\prime}
$$

and

$$
I_{k}^{*}\left(\tau_{0},-\mu\right)=\rho_{2}^{s} I_{k}^{*}\left(\tau_{0}, \mu\right)+2 \rho_{2}^{d} \int_{0}^{1} I_{k}^{*}\left(\tau_{0}, \mu^{\prime}\right) \mu^{\prime} d \mu^{\prime}
$$

for $\mu \in[0,1]$. In addition, the homogeneous problem is defined by

$$
\mu \frac{\partial}{\partial \tau} I_{h}^{*}(\tau, \mu)+I_{h}^{*}(\tau, \mu)=\frac{\varpi}{2} \sum_{l=0}^{L} \beta_{l} P_{l}(\mu) \int_{-1}^{1} P_{l}\left(\mu^{\prime}\right) I_{h}^{*}\left(\tau, \mu^{\prime}\right) d \mu^{\prime}
$$

for $\tau \in\left(0, \tau_{0}\right)$ and $\mu \in[-1,1]$,

$$
I_{h}^{*}(0, \mu)=\epsilon_{1} \Theta_{1}^{4}
$$

and

$$
I_{h}^{*}\left(\tau_{0},-\mu\right)=\epsilon_{2} \Theta_{2}^{4} .
$$

We can solve Eq. (11) to find

$$
\Theta(\tau)=\Theta_{h}(\tau)+\sum_{k=0}^{K} a_{k} \Theta_{k}(\tau)
$$

where

$$
\Theta_{h}(\tau)=\Theta_{1}+\frac{\tau}{\tau_{0}}\left(\Theta_{2}-\Theta_{1}\right)+\frac{1}{4 \pi N_{c}}\left(\int_{0}^{\tau} q_{r, h}^{*}(x) d x-\frac{\tau}{\tau_{0}} \int_{0}^{\tau_{0}} q_{r, h}^{*}(x) d x\right)
$$


and, for $k=0,1,2, \ldots, K$,

$$
\Theta_{k}(\tau)=\frac{1}{4 \pi N_{c}}\left(\int_{0}^{\tau} q_{r, k}^{*}(x) d x-\frac{\tau}{\tau_{0}} \int_{0}^{\tau_{0}} q_{r, k}^{*}(x) d x\right)
$$

with

$$
q_{r, h}^{*}(\tau)=2 \pi \int_{-1}^{1} I_{h}^{*}(\tau, \mu) \mu d \mu
$$

and, for $k=0,1,2, \ldots, K$,

$$
q_{r, k}^{*}(\tau)=2 \pi \int_{-1}^{1} I_{k}^{*}(\tau, \mu) \mu d \mu
$$

As we can use, for example, the spherical harmonics method (see Ref. 1) to establish the temperature fuctions $\Theta_{h}(\tau)$ and $\Theta_{k}(\tau)$, for $k=0,1,2, \ldots, K$, we have only to compute the unknown coefficients $\left\{a_{k}\right\}$ to complete our solution. It is clear, from Eqs. (15), (16) and (22), that we can write

$$
\sum_{k=0}^{K} a_{k} \phi_{k}\left(\tau / \tau_{0}\right)=(1-\varpi)\left[\Theta_{h}(\tau)+\sum_{k=0}^{K} a_{k} \Theta_{k}(\tau)\right]^{4}
$$

We let $\zeta_{0}, \zeta_{1}, \zeta_{2}, \ldots, \zeta_{M}$, where $M=(K-1) / 2$, denote the knots used to define our spline functions and evaluate $\mathrm{Eq} .(25)$ and the derivative of $\mathrm{Eq} .(25)$ at each of the points $\tau=\zeta_{\alpha} \tau_{0}$, for $\alpha=0,1,2, \ldots, M$, to obtain the system of nonlinear algebraic equations

$$
\sum_{k=0}^{K} a_{k} \phi_{k}\left(\zeta_{\alpha}\right)=(1-\varpi)\left[\Theta_{h}\left(\zeta_{\alpha} \tau_{0}\right)+\sum_{k=0}^{K} a_{k} \Theta_{k}\left(\zeta_{\alpha} \tau_{0}\right)\right]^{4}
$$

and

$$
\begin{aligned}
\left.\sum_{k=0}^{K} a_{k} \frac{d}{d \tau} \phi_{k}\left(\tau / \tau_{0}\right)\right|_{\tau=\zeta_{\alpha} \tau_{0}} & = \\
& \left.4(1-\varpi)\left[\Theta_{h}\left(\zeta_{\alpha} \tau_{0}\right)+\sum_{k=0}^{K} a_{k} \Theta_{k}\left(\zeta_{\alpha} \tau_{0}\right)\right]^{3} \frac{d}{d \tau}\left[\Theta_{h}(\tau)+\sum_{k=0}^{K} a_{k} \Theta_{k}(\tau)\right]\right|_{\tau=\zeta_{\alpha} \tau_{0}}
\end{aligned}
$$

which we consider to define to required constants $\left\{a_{k}\right\}$. If we let the components of a $(K+1)$-vector $\mathbf{F}(\mathbf{a})$, where the vector a has components $a_{0}, a_{1}, \ldots, a_{K}$, be defined, for $\alpha=0,1, \ldots, M$, by

$$
F_{\alpha}(\mathbf{a})=\sum_{k=0}^{K} a_{k} \phi_{k}\left(\zeta_{\alpha}\right)-(1-\varpi)\left[\Theta_{h}\left(\zeta_{\alpha} \tau_{0}\right)+\sum_{k=0}^{K} a_{k} \Theta_{k}\left(\zeta_{\alpha} \tau_{0}\right)\right]^{4}
$$

and

$$
\begin{aligned}
F_{M+1+\alpha}(\mathbf{a})= & \left.\sum_{k=0}^{K} a_{k} \frac{d}{d \tau} \phi_{k}\left(\tau / \tau_{0}\right)\right|_{\tau=\zeta_{\alpha} \tau_{0}} \\
& \quad-\left.4(1-\varpi)\left[\Theta_{h}\left(\zeta_{\alpha} \tau_{0}\right)+\sum_{k=0}^{K} a_{k} \Theta_{k}\left(\zeta_{\alpha} \tau_{0}\right)\right]^{3} \frac{d}{d \tau}\left[\Theta_{h}(\tau)+\sum_{k=0}^{K} a_{k} \Theta_{k}(\tau)\right]\right|_{\tau=\zeta_{\alpha} \tau_{0}}
\end{aligned}
$$


then we can express the Newton iteration scheme as

$$
\mathbf{a}_{j+1}=\mathbf{a}_{j}+\mathbf{J}^{-1}\left(\mathbf{a}_{j}\right) \mathbf{F}\left(\mathbf{a}_{j}\right)
$$

for $j=0,1, \ldots$ Here the Jacobian matrix is given by

$$
\mathbf{J}(\mathbf{a})=\left(\frac{\partial}{\partial a_{0}} \mathbf{F}(\mathbf{a}), \frac{\partial}{\partial a_{1}} \mathbf{F}(\mathbf{a}), \ldots, \frac{\partial}{\partial a_{K}} \mathbf{F}(\mathbf{a})\right)
$$

For our initial approximation we take $\mathbf{a}_{0}$ as obtained by using a linear temperature distribution to define a first approximation of the source term of $S(\tau)$.

Assuming that we have found a converged result from Eq. (28), we can use Eqs. (15) and (16) to find the required normalized temperature distribution, viz.

$$
\Theta(\tau)=\left[\left(\frac{1}{1-\varpi}\right) \sum_{k=0}^{K} a_{k} \phi_{k}\left(\tau / \tau_{0}\right)\right]^{1 / 4} .
$$

We can then differentiate Eq. (30) and compute the normalized conductive heat flux from

$$
Q_{c}(\tau)=-\frac{d}{d \tau} \Theta(\tau)
$$

The normalized radiative heat flux can be evaluated from

$$
Q_{r}(\tau)=\frac{1}{2 N_{c}}\left[q_{r, h}^{*}(\tau)+\sum_{k=0}^{K} a_{k} q_{r, k}^{*}(\tau)\right],
$$

and finally the normalized total heat flux is given by

$$
Q(\tau)=Q_{c}(\tau)+Q_{r}(\tau)
$$

\section{Numerical Results}

To have a specific scattering law for testing our solution technique, and to avoid having to provide a table of the scattering law coefficients $\left\{\beta_{l}\right\}$, we use here the binomial scattering law ${ }^{5}$

$$
p(\cos \Theta)=\frac{L+1}{2^{L}}(1+\cos \Theta)^{L}
$$

which can be represented exactly with $L+1$ Legendre coefficients that can be computed with $\beta_{0}=1$ and ${ }^{6}$

$$
\beta_{l}=\left(\frac{2 l+1}{2 l-1}\right)\left(\frac{L+1-l}{L+1+l}\right) \beta_{l-1}
$$


To make available some numerical results that have been obtained with the Newton method of iteration discussed here, we consider the four test problems defined in Table 1 . As we wish to solve problems here that we were unable to solve with the iteration procedure used in Ref. 1 , we elect to use here $N_{e}=0.00001$ and to keep all of the other defining parameters the same as for the related problems solved in Ref. 1 for the case $N_{c}=0.05$.

Our converged results for the normalized temperature distribution and the normalized heat fluxes are given in Tables 2-5. Having varied the order of the $P_{N}$ approximation, the number of Hermite splines used and the number of Gauss points used to evaluate some integrals over the spline functions, we have some confidence that the results given in Tables $2-5$ are correct to within one unit in the last digit given.

To conclude this work we would like to record a few remarks. In regard to the existence and uniqueness issues mentioned in Ref. 1, we note that Kelley ${ }^{4}$ has provided existence and uniqueness theorems that apply to our problem for the case of isotropic scattering and non-reflecting boundaries; we note also that work aimed at extending those theorems is continuing. ${ }^{4}$ Also, as we have no proof that the Newton iteration scheme we use actually converges, we can only conjecture that the results given in Tables 2-7 are correct.

Finally, we must conclude that Newton's method of iteration is, in general, vastly superior to the simple iteration scheme used in Ref. 1, and so it is clear that Newton's method can also be used to extend the class of problems solved for spheres and cylinders in Refs. 7 and 8 .

\section{Acknowledgement}

The author wishes to express his appreciation to C. T. Kelley for several helpful discussions concerning this work. 


\section{References}

1. C. E. Siewert and J. R. Thomas, Jr., JQSRT 45, 273 (1991).

2. M. Benassi, R. D. M. Garcia, A. H. Karp and C. E. Siewert, Astrophys. J. 280, 853 (1984).

3. M. N. Özışık, Radiative Transfer and Interaction with Conduction and Convection, John Wiley \& Sons, New York (1973).

4. C. T. Kelley, private communication and preprint (1994).

5. H. G. Kaper, J. K. Shultis and J. G. Veninga, J. Comp. Phys. 6, 288 (1970).

6. N. J. McCormick and R. Sanchez, J. Math. Phys. 22, 199 (1981).

7. C. E. Siewert and J. R. Thomas, Jr., JQSRT 46, 63 (1991).

8. C. E. Siewert and J. R. Thomas, Jr., JQSRT 48, 227 (1991). 
Table 1. Physical Data for Different Problems

\begin{tabular}{|ccccccccccccc|}
\hline Problem & $\epsilon_{1}$ & $\epsilon_{2}$ & $\rho_{1}^{s}$ & $\rho_{2}^{s}$ & $\rho_{1}^{d}$ & $\rho_{2}^{d}$ & $\Theta_{1}$ & $\Theta_{2}$ & $\varpi$ & $\tau_{0}$ & $N_{c}$ & $L$ \\
\hline 1 & 1.0 & 1.0 & 0.0 & 0.0 & 0.0 & 0.0 & 1.0 & 0.5 & 0.9 & 1.0 & 0.00001 & 0 \\
2 & 0.7 & 0.6 & 0.1 & 0.3 & 0.2 & 0.1 & 1.0 & 0.5 & 0.9 & 3.0 & 0.00001 & 0 \\
3 & 1.0 & 1.0 & 0.0 & 0.0 & 0.0 & 0.0 & 1.0 & 0.5 & 0.95 & 1.0 & 0.00001 & 299 \\
4 & 0.8 & 0.8 & 0.1 & 0.1 & 0.1 & 0.1 & 1.0 & 0.5 & 0.99 & 3.0 & 0.00001 & 299 \\
\hline
\end{tabular}

centerlineTable 2. Normalized Temperature Distribution and Heat Fluxes for Problem 1

\begin{tabular}{|lllll|}
\hline$\tau / \tau_{0}$ & \multicolumn{1}{c}{$\Theta(\tau)$} & \multicolumn{1}{c}{$Q_{c}(\tau)$} & $Q_{r}(\tau)$ & $Q(\tau)$ \\
\hline 0.00 & 1.0 & $1.192(1)$ & $1.29680(4)$ & $1.29799(4)$ \\
0.01 & $9.45293(-1)$ & 2.024 & $1.29779(4)$ & $1.29799(4)$ \\
0.05 & $9.27635(-1)$ & $1.787(-1)$ & $1.29797(4)$ & $1.29799(4)$ \\
0.10 & $9.19142(-1)$ & $1.64429(-1)$ & $1.29797(4)$ & $1.29799(4)$ \\
0.20 & $9.03101(-1)$ & $1.58610(-1)$ & $1.29797(4)$ & $1.29799(4)$ \\
0.30 & $8.87169(-1)$ & $1.60829(-1)$ & $1.29797(4)$ & $1.29799(4)$ \\
0.40 & $8.70808(-1)$ & $1.66932(-1)$ & $1.29797(4)$ & $1.29799(4)$ \\
0.50 & $8.53678(-1)$ & $1.76206(-1)$ & $1.29797(4)$ & $1.29799(4)$ \\
0.60 & $8.35449(-1)$ & $1.89059(-1)$ & $1.29797(4)$ & $1.29799(4)$ \\
0.70 & $8.15700(-1)$ & $2.06973(-1)$ & $1.29797(4)$ & $1.29799(4)$ \\
0.80 & $7.93766(-1)$ & $2.33741(-1)$ & $1.29797(4)$ & $1.29799(4)$ \\
0.90 & $7.68273(-1)$ & $2.82207(-1)$ & $1.29796(4)$ & $1.29799(4)$ \\
0.95 & $7.52599(-1)$ & $3.916(-1)$ & $1.29795(4)$ & $1.29799(4)$ \\
0.99 & $6.64184(-1)$ & 9.358 & $1.29706(4)$ & $1.29799(4)$ \\
1.00 & $5.0(-1)$ & $2.559(1)$ & $1.29543(4)$ & $1.29799(4)$ \\
\hline
\end{tabular}

Table 3. Normalized Temperature Distribution and Heat Fluxes for Problem 2

\begin{tabular}{|lllll|}
\hline$\tau / \tau_{0}$ & \multicolumn{1}{c}{$\Theta(\tau)$} & \multicolumn{1}{c}{$Q_{c}(\tau)$} & $Q_{r}(\tau)$ & $Q(\tau)$ \\
\hline 0.00 & 1.0 & 9.36 & $5.29641(3)$ & $5.30577(3)$ \\
0.01 & $9.48894(-1)$ & $1.10(-1)$ & $5.30566(3)$ & $5.30577(3)$ \\
0.05 & $9.40977(-1)$ & $5.91125(-2)$ & $5.30571(3)$ & $5.30577(3)$ \\
0.10 & $9.32452(-1)$ & $5.53844(-2)$ & $5.30571(3)$ & $5.30577(3)$ \\
0.20 & $9.16047(-1)$ & $5.46797(-2)$ & $5.30571(3)$ & $5.30577(3)$ \\
0.30 & $8.99417(-1)$ & $5.64179(-2)$ & $5.30571(3)$ & $5.30577(3)$ \\
0.40 & $8.82090(-1)$ & $5.92490(-2)$ & $5.30571(3)$ & $5.30577(3)$ \\
0.50 & $8.63780(-1)$ & $6.29676(-2)$ & $5.30571(3)$ & $5.30577(3)$ \\
0.60 & $8.44207(-1)$ & $6.77151(-2)$ & $5.30570(3)$ & $5.30577(3)$ \\
0.70 & $8.23002(-1)$ & $7.39731(-2)$ & $5.30569(3)$ & $5.30577(3)$ \\
0.80 & $7.99552(-1)$ & $8.30248(-2)$ & $5.30568(3)$ & $5.30577(3)$ \\
0.90 & $7.72487(-1)$ & $9.96011(-2)$ & $5.30567(3)$ & $5.30577(3)$ \\
0.95 & $7.56331(-1)$ & $1.18386(-1)$ & $5.30565(3)$ & $5.30577(3)$ \\
0.99 & $7.337(-1)$ & $9.54(-1)$ & $5.30481(3)$ & $5.30577(3)$ \\
1.00 & $5.0(-1)$ & $2.54(1)$ & $5.28040(3)$ & $5.30577(3)$ \\
\hline
\end{tabular}


Table 4. Normalized Temperature Distribution and Heat Fluxes for Problem 3

\begin{tabular}{|lllll|}
\hline$\tau / \tau_{0}$ & \multicolumn{1}{c}{$\Theta(\tau)$} & \multicolumn{1}{c}{$Q_{c}(\tau)$} & \multicolumn{1}{c}{$Q_{r}(\tau)$} & $Q(\tau)$ \\
\hline 0.00 & 1.0 & $1.527(1)$ & $2.20768(4)$ & $2.20921(4)$ \\
0.01 & $9.13550(-1)$ & 4.405 & $2.20877(4)$ & $2.20921(4)$ \\
0.05 & $8.74381(-1)$ & $1.059(-1)$ & $2.20920(4)$ & $2.20921(4)$ \\
0.10 & $8.71112(-1)$ & $5.34518(-2)$ & $2.20920(4)$ & $2.20921(4)$ \\
0.20 & $8.66268(-1)$ & $4.51222(-2)$ & $2.20920(4)$ & $2.20921(4)$ \\
0.30 & $8.61925(-1)$ & $4.21786(-2)$ & $2.20920(4)$ & $2.20921(4)$ \\
0.40 & $8.57770(-1)$ & $4.11385(-2)$ & $2.20920(4)$ & $2.20921(4)$ \\
0.50 & $8.53659(-1)$ & $4.12457(-2)$ & $2.20920(4)$ & $2.20921(4)$ \\
0.60 & $8.49488(-1)$ & $4.23702(-2)$ & $2.20920(4)$ & $2.20921(4)$ \\
0.70 & $8.45143(-1)$ & $4.47814(-2)$ & $2.20920(4)$ & $2.20921(4)$ \\
0.80 & $8.40457(-1)$ & $4.94963(-2)$ & $2.20920(4)$ & $2.20921(4)$ \\
0.90 & $8.35032(-1)$ & $6.17824(-2)$ & $2.20920(4)$ & $2.20921(4)$ \\
0.95 & $8.29743(-1)$ & $2.826(-1)$ & $2.20918(4)$ & $2.20921(4)$ \\
0.99 & $6.99929(-1)$ & $1.275(1)$ & $2.20793(4)$ & $2.20921(4)$ \\
1.00 & $5.0(-1)$ & $2.872(1)$ & $2.20633(4)$ & $2.20921(4)$ \\
\hline
\end{tabular}

Table 5. Normalized Temperature Distribution and Heat Fluxes for Problem 4

\begin{tabular}{|lllll|}
\hline$\tau / \tau_{0}$ & \multicolumn{1}{c}{$\Theta(\tau)$} & \multicolumn{1}{c}{$Q_{c}(\tau)$} & $Q_{r}(\tau)$ & $Q(\tau)$ \\
\hline 0.00 & 1.0 & 6.993 & $1.49489(4)$ & $1.49559(4)$ \\
0.01 & $8.98702(-1)$ & 1.354 & $1.49546(4)$ & $1.49559(4)$ \\
0.05 & $8.70402(-1)$ & $2.298(-2)$ & $1.49559(4)$ & $1.49559(4)$ \\
0.10 & $8.67735(-1)$ & $1.54505(-2)$ & $1.49559(4)$ & $1.49559(4)$ \\
0.20 & $8.63661(-1)$ & $1.23167(-2)$ & $1.49559(4)$ & $1.49559(4)$ \\
0.30 & $8.60163(-1)$ & $1.11720(-2)$ & $1.49559(4)$ & $1.49559(4)$ \\
0.40 & $8.56891(-1)$ & $1.07183(-2)$ & $1.49559(4)$ & $1.49559(4)$ \\
0.50 & $8.53692(-1)$ & $1.06676(-2)$ & $1.49559(4)$ & $1.49559(4)$ \\
0.60 & $8.50456(-1)$ & $1.09659(-2)$ & $1.49559(4)$ & $1.49559(4)$ \\
0.70 & $8.47069(-1)$ & $1.17040(-2)$ & $1.49559(4)$ & $1.49559(4)$ \\
0.80 & $8.43356(-1)$ & $1.32407(-2)$ & $1.49559(4)$ & $1.49559(4)$ \\
0.90 & $8.38908(-1)$ & $1.71475(-2)$ & $1.49559(4)$ & $1.49559(4)$ \\
0.95 & $8.35678(-1)$ & $3.741(-2)$ & $1.49559(4)$ & $1.49559(4)$ \\
0.99 & $7.40963(-1)$ & 4.198 & $1.49517(4)$ & $1.49559(4)$ \\
1.00 & $5.0(-1)$ & $1.308(1)$ & $1.49429(4)$ & $1.49559(4)$ \\
\hline
\end{tabular}

